\title{
Spread of Multidrug-Resistant Rhodococcus equi, United States
}

\author{
Sonsiray Álvarez-Narváez, Steeve Giguère, ${ }^{1}$ Noah Cohen, Nathan Slovis, José A. Vázquez-Boland
}

\begin{abstract}
Multidrug resistance has been detected in the animal and zoonotic human pathogen Rhodococcus equi after mass macrolide/rifampin antibioprophylaxis in endemically affected equine farms in the United States. Multidrug-resistant (MDR) R. equi emerged upon acquisition of pRErm46, a conjugative plasmid conferring resistance to macrolides, lincosamides, streptogramins, and, as we describe, tetracycline. Phylogenomic analyses indicate that the increasing prevalence of MDR $R$. equi since it was first documented in 2002 is caused by a clone, $R$. equi 2287 , attributable to coselection of pRErm46 with a chromosomal $r p o B^{\mathrm{S531F}}$ mutation driven by macrolide/rifampin therapy. pRErm46 spillover to other $R$. equi genotypes has given rise to a novel MDR clone, G2016, associated with a distinct $r p o B^{\mathrm{S} 531 Y}$ mutation. Our findings illustrate that overuse of antimicrobial prophylaxis in animals can generate MDR pathogens with zoonotic potential. MDR $R$. equi and pRErm46mediated resistance are currently disseminating in the United States and are likely to spread internationally through horse movements.
\end{abstract}

$R$ hodococcus equi is a soilborne facultative intracellular actinobacterium that causes pyogranulomatous infections in multiple animal species, including humans. Rhodococcal infection is particularly severe in young foals and immunocompromised persons, in whom it typically manifests as a life-threatening purulent bronchopneumonic disease (1-3). R. equi is able to colonize equids, pigs, and ruminants through 3 different host-specific virulence plasmid types (designated pVAPA, pVAPB, and pVAPN) (4). Analysis of the virulence plasmids carried by the isolates and comparison of genomic profiles indicate that human R. equi infections originate from animals (4-6).

Author affiliations: University of Georgia, Athens, Georgia, USA

(S. Álvarez-Narváez, S. Giguère); Texas A\&M University, College Station, Texas, USA (N. Cohen); Hagyard Equine Medical Institute, Lexington, Kentucky, USA (N. Slovis); University of Edinburgh, Edinburgh, Scotland, UK (J.A. Vázquez-Boland)

DOI: https://doi.org/10.3201/eid2702.203030
R. equi is highly prevalent in horse-breeding farms worldwide (7). For decades, the standard treatment for $R$. equi pneumonia in foals has been a combination macrolide/rifampin therapy (8). In the absence of effective preventive methods, many horse-breeding farms rely on early ultrasonographic detection of infected foals and initiation of macrolide/rifampin prophylaxis before clinical manifestation of the disease (9). In the United States, where foal rhodococcosis is often endemic, implementation of this practice has been linked to the emergence of dual macrolide/rifampin-resistant $\left(\mathrm{MR}^{\mathrm{R}}\right)$ R. equi (10-12). First detected in the late 1990s, R. equi $\mathrm{MR}^{\mathrm{R}}$ isolates are increasingly prevalent (11-14), posing a substantial problem because no clinically proven therapeutic alternative is currently available for the treatment of affected foals (8). The $\mathrm{MR}^{\mathrm{R}}$ isolates also represent a potential hazard to human health because of the risk for zoonotic transmission.

We recently determined that the emerging $M^{R}$ phenotype among $R$. equi equine isolates was linked to a novel methyltransferase gene, erm(46), which confers cross-resistance to macrolides, lincosamides, and streptogramins (MLS ${ }^{R}$ phenotype) (13). erm(46) is part of a $6.9-\mathrm{kb}$ transposable element, TnRErm46, which is carried by the conjugative resistance plasmid pRErm46 (15). Upon pRErm46 acquisition, TnRErm 46 stabilizes itself in $R$. equi by transposing to the host genome, including the conjugative virulence plasmid pVAPA. Despite its high potential for horizontal spread, we found that pRErm46/TnRErm46 was restricted to a specific $R$. equi clone, designated 2287, likely because of co-selection with a chromosomal rifampin-resistance $r p o B^{\mathrm{S} 531 \mathrm{~F}}$ mutation in response to macrolide/rifampin therapy (15).

We identified the multidrug-resistant (MDR) $R$. equi 2287 clone by analyzing isolates collected during 2002-2011 (15). Here, we investigate the spread of the erm(46) determinant in a contemporary sample 
of macrolide-resistant isolates and horizontal spread of pRErm46/TnRErm46, leading to emergence of a further MDR $R$. equi clone associated with a novel $r p o B^{\mathrm{S} 531 Y}$ mutation.

\section{Materials and Methods}

\section{Bacteria}

We sequenced the genomes of a random selection of 30 macrolide-resistant and 18 macrolide-susceptible $R$. equi equine clinical strains recovered from pneumonic foals in 5 US states (Florida, Kentucky, Louisiana, New York, and Texas) during 2012-2017 (Appendix Table 1, https://wwwnc.cdc.gov/EID/ article/27/2/20-3030-App1.pdf). Whenever possible, at least 1 strain from each category was chosen for each year and US state. The strains from Louisiana were a random collection of 10 convenience-sampled isolates from a single farm. All strains were routinely grown in brain-heart infusion medium (BD, https:// www.bd.com) for $48 \mathrm{~h}$ at $37^{\circ} \mathrm{C}$. Detection of the erm(46) gene by PCR was performed as previously described $(13,15)$.

\section{Antimicrobial Susceptibility Testing}

Susceptibility tests were performed at the Hagyard Equine Medical Institute diagnostic laboratory (Lexington, Kentucky, USA), Texas A\&M Veterinary Medical Diagnostic Laboratory (College Station, Texas, USA), and University of Georgia Veterinary Diagnostic Laboratory (Athens, Georgia, USA) according to Clinical and Laboratory Standards Institute (CLSI) guidelines (https://clsi.org). In the absence of specific disk susceptibility interpretive criteria for R. equi, CLSI guidelines for Staphylococcus aureus were used in accordance with routine practices in veterinary diagnostic laboratories $(11,16)$. MICs were determined in tryptone soy agar medium by using Etest strips (bioMérieux, https://www.biomerieux.com) according to the manufacturer's recommendations (16). Staphylococcus aureus ATCC 29213 was used as a control in all susceptibility tests.

\section{Genome Sequencing and Phylogenetic Analysis}

We extracted bacterial genomic DNA by using DNeasy UltraClean Microbial Kit (QIAGEN, https://www.qiagen.com) following the manufacturer's instructions. DNA quality (optical density 260/280, ratio 1.8:2) and concentration $(>1 \mu \mathrm{g})$ of each gDNA sample were verified by using a NanoDrop apparatus (Thermo Fisher Scientific, https:/ / www.thermofisher.com). Single-molecule real-time long-read DNA sequencing was performed at Duke
Center for Genomic and Computational Biology (Duke University, Durham, North Carolina, USA). SMRTbell Template Prep Kit 2.0 was used for library preparation of 4-6-kb insert for 8 multiplexed bacterial samples. Samples were run on a PacBio Sequel II system (Pacific Bioscience, https:/ / www.pacb.com). Genomes were assembled de novo by using Canu version 1.9 (17). Whole-genome phylogenetic analysis was performed with ParSNP in the Harvest suite, designed for single-nucleotide polymorphism analysis between closely related species or strains ( $\geq 97 \%$ average nucleotide identity) (18). The program uses FastTree 2 (19) to build approximately maximumlikelihood trees from core-genome single-nucleotide polymorphisms. Trees were visualized in FigTree 1.4.4 (http://tree.bio.ed.ac.uk/software/figtree). Principal component analysis was performed by feeding VCF files extracted from ParSNP alignments to ggfortify package in $\mathrm{R}$ software version 3.6.1 (https://cran.r-project.org/web/packages/ ggfortify/index.html).

\section{Statistical Analysis}

Statistical significance of tetracycline susceptibility data was determined by $\chi^{2}$ test and Student t-test. All tests were conducted using Prism software version 8 (https://www.graphpad.com).

\section{Results}

The 30 macrolide-resistant $R$. equi genome sequences determined in this study were subjected to phylogenetic analysis alongside a sample of 18 susceptible isolates from the same period and geographic origins to examine their relationships. The macrolideresistant isolates had previously tested positive to erm(46) by PCR and most ( $\mathrm{n}=22,73 \%$ ) were also resistant to rifampin (MR ${ }^{\mathrm{R}}$ phenotype). Of note, 8 of the 2012-2017 R. equi isolates examined here were macrolide-only-resistant (Appendix Table 1); to date, dual $M^{R}$ resistance had been invariably observed $(10,11,13,15)$. We also included in our analysis Illumina whole-genome assemblies from 22 equine isolates characterized in our earlier study $(n=16$ belonging to the 2287 clone, $n=6$ control susceptible isolates) and 23 macrolide-susceptible strains representative of the global genomic diversity of $R$. equi (20). Figure 1 shows the core-genome phylogeny of the 93 R. equi strains.

\section{Clonal Spread of MDR R. equi 2287}

Of 22 in total, 20 (91\%) of the new MR ${ }^{\mathrm{R}}$ isolates clustered together at short genetic distances with the previously characterized MDR 2287 isolates, indicating 


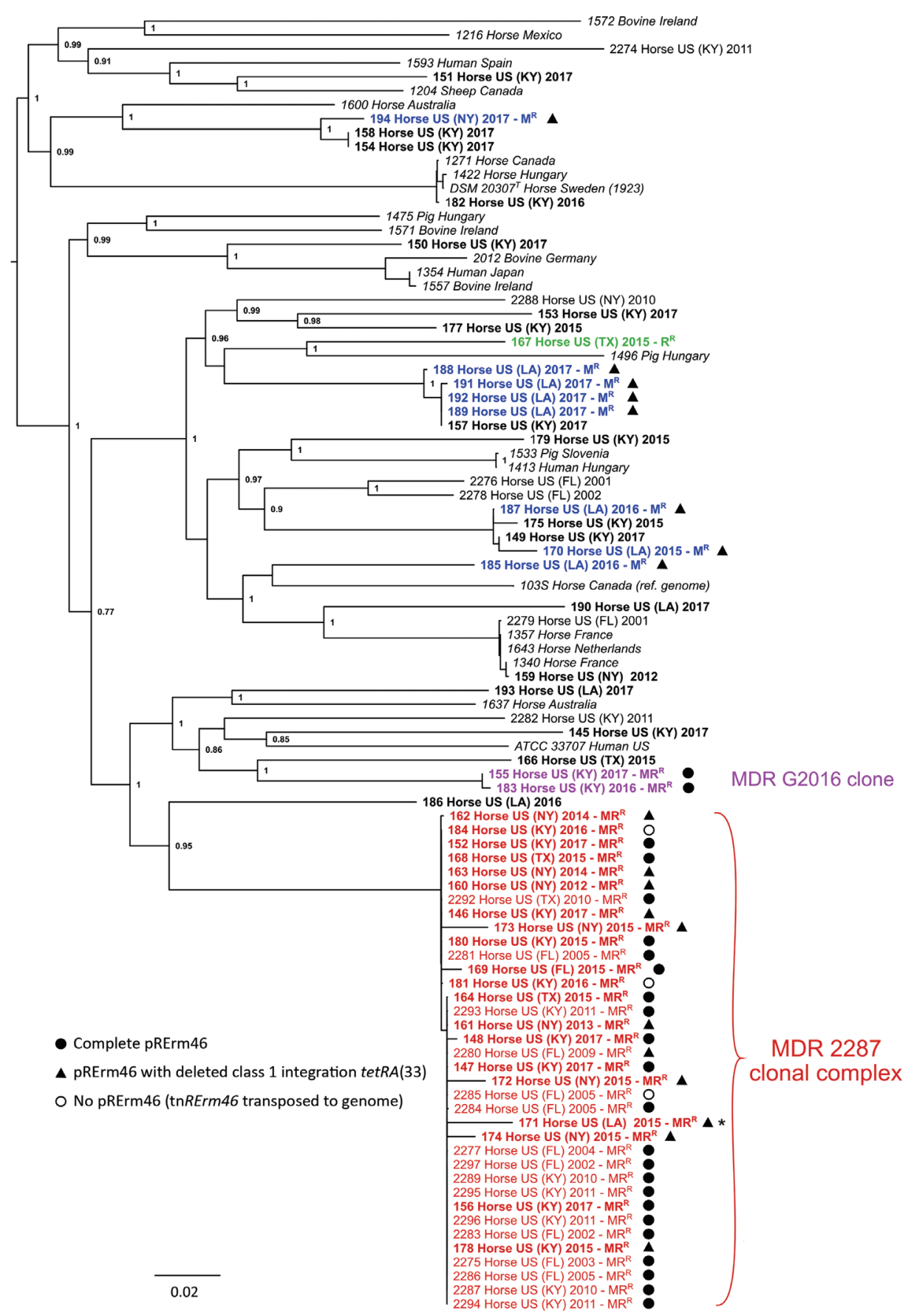

Figure 1. Spread and phylogenetic relationships of MDR Rhodococcus equi, United States. Phylogenetic tree of $93 R$. equi isolates based on core-genome single-nucleotide polymorphism analysis by using ParSNP (18). The genomes analyzed are from 58 erm(46)-positive $M^{R}$ isolates, 24 control-susceptible isolates from same period and geographic origins, and 23 isolates representative of the genomic diversity of $R$. equi, including the reference genome 103S (33) and the type strain DSM $20307^{\top}$ (Appendix Table 1, https://wwwnc. cdc.gov/EID/article/27/2/203030-App1.pdf). Tip labels show year of collection and resistance phenotype for the 2001-2017 equine clinical isolates analyzed (the 50 genomes determined in this study are shown in bold, and other genomes are from previous study [15]). Red indicates MDR 2287 clonal complex, violet indicates novel MDR G2016 clone, blue indicates genetically diverse $M^{R}$ isolates recovered from a farm in Louisiana during 2015-2017 (MDR 2287 isolate from which they likely acquired the pRErm46 plasmid is indicated by an asterisk), and green indicates an $\mathrm{R}^{\mathrm{R}}$ isolate $(r p o B \mathrm{~S} 531 \mathrm{~K}$ mutation). pRErm46 carriage status is indicated by symbols. Tree graph constructed with FigTree (http://tree.bio.ed.ac.uk/ software/figtree). MDR, multidrugresistant; $\mathrm{M}^{\mathrm{R}}$, macrolide-resistant;

Complete pRErm46

pRErm46 with deleted class 1 integration tetRA(33)

they correspond to the same clonal population (Figure 1). Accordingly, all of the newly sequenced $M^{R}$ strains possessed the $r p o B^{\mathrm{S531F}}$ mutation unique to the 2287 clone. Of those, 2 had lost the pRErm46 plasmid and only carried the TnRErm 46 transposon (Figure 1), as previously observed in 1 of the 18 isolates from the 2002-2011 series (15). Collection times and locations encompassed the entire 2012-2017 period and the 5 US states for the MDR 2287 clonal popula- tion. The lack of spatial-temporal circumscription of MDR 2287 in the analyzed sample is illustrated by a principal components analysis in which the only grouping factor for the $93 R$. equi isolates included in this study is the genetic background of the 2287 clone (Figure 2).

We repeated the phylogenomic analysis with the 36 R. equi 2287 sequences from 2002-2017 to assess the microevolution of the clone. This analysis 

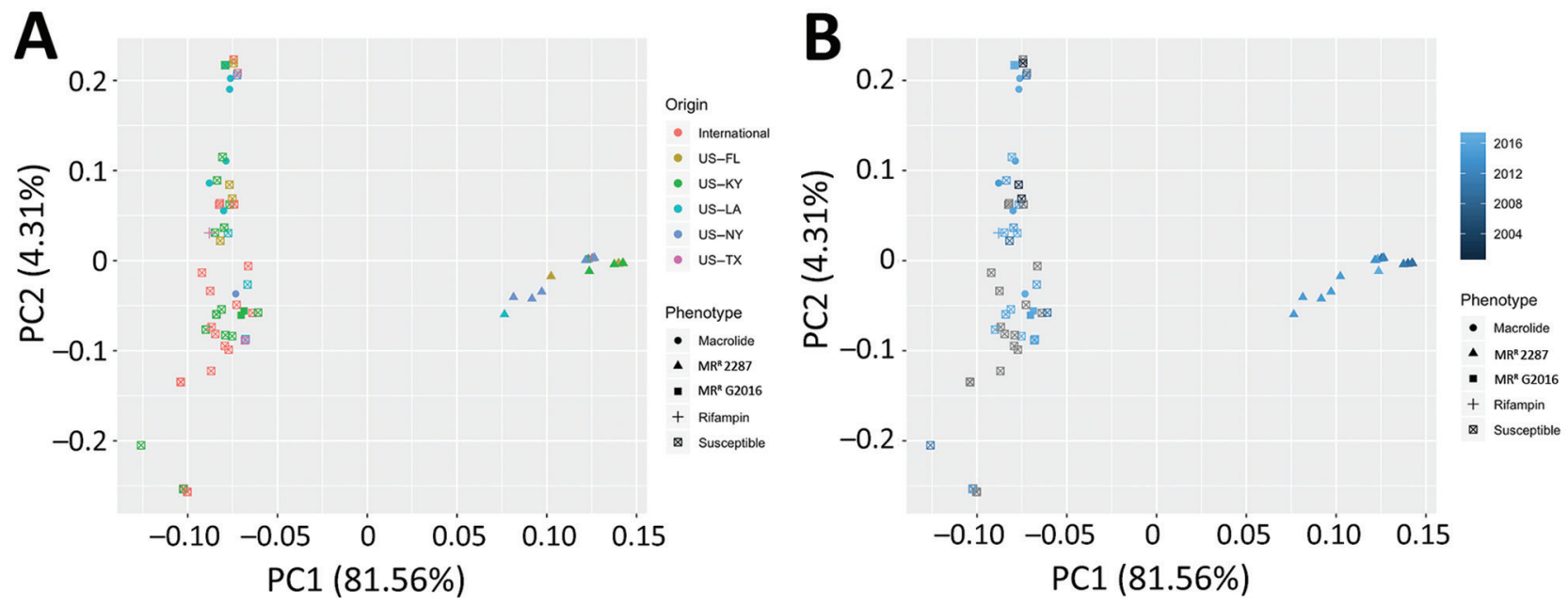

Figure 2. Lack of spatial-temporal circumscription of multidrug-resistant Rhodococcus equi clone 2287, United States. Principal component analysis plot was constructed on the basis of the single-nucleotide polymorphism variant calls obtained in the phylogenetic analysis. Isolates are identified by resistance group and color-coded by geographic origin $(A)$ or year of isolation $(B)$. MR ${ }^{R}$, dual macrolide/rifampin resistant; PC1, principal component 1; PC2, principal component 2.

revealed that MDR 2287 had diversified into 3 major radiations (Figure 3 ), consistent with the clonal structure of $R$. equi evolution (20). Of note, 1 of these subclades gathered 11 of the 16 older isolates from 2002-2011, all originating from Florida or Kentucky. The remaining 5 older isolates were distributed in the 2 other subclades in which strains were grouped independently of year of collection or geographic origin. This distribution suggests a pattern of spread defined by the diversification of MDR 2287 into subclonal lineages and increasing exchange between horse farms of a progressively diverse clonal population.

\section{Dissemination of pRErm46 and Emergence of Novel MDR $R$. equi Clone}

Ten macrolide-resistant isolates also carried pRErm46 but did not belong to the MDR 2287 clone and were genetically diverse. Most appeared as singletons interspersed among the different lines of descent in the R. equi tree (Figure 1). In this group, 8 strains corresponded to the previously mentioned macrolide-only-resistant isolates (i.e., rifampin susceptible, no rpoB mutation; MIC <0.125-1.25 $\mu \mathrm{g} / \mathrm{mL}$ ). All but 1 of these isolates originated from the same farm in Louisiana in which an MDR 2287 isolate (no. 171) was recovered during the same period. This circumstance suggests a scenario in which the entry of MDR 2287 into this farm resulted in the conjugal spread of pRErm46 to different members of the heterogeneous $R$. equi populations that are typically found colonizing horse-breeding environments, or even individual animals within the same farm $(21,22)$.

Of interest, 2 of the non-2287 macrolide-resistant isolates, numbers 155 (recovered in Kentucky in 2017) and 183 (recovered in Kentucky in 2016), were also resistant to rifampin (MIC $>32 \mu \mathrm{g} / \mathrm{mL}$ ) (Figure $1)$. These 2 nearly genomically identical $\mathrm{MR}^{\mathrm{R}}$ strains carried the pRErm 46 plasmid and a chromosomal rpoB mutation, Ser531Tyr (Escherichia coli numbering), distinct from that in MDR 2287 and novel in $R$. equi. Both $\mathrm{MR}^{\mathrm{R}}$ isolates constitute a new emerging MDR R. equi clone, first detected in 2016, which we designated G2016.

Collectively, these data indicate that the pRErm46 macrolide-resistance plasmid, until now unique to the 2287 clone, has recently undergone horizontal transfer events to multiple $R$. equi genotypes. These transfers gave rise to novel MDR clones when associated with an rpoB mutation.

\section{pRErm46 Variability and Tetracycline Resistance}

pRErm46 also harbors a class 1 integron (C1I) with a tetR-tet $A$ cassette encoding a putative tetracycline efflux pump homologous to TetA(33) from the corynebacterial plasmid pTET3 $(15,23)$. TetA efflux pumps are often carried by transposons and are one of the most prevalent tetracycline-resistance mechanisms (24). Both the C1I and tetRA determinant from pRErm46 are virtually identical to those from pTET3, including flanking IS6100 insertion sequences (15). Blast alignments revealed that the C1I-tetRA(33) region was deleted in 17 of the $43(40 \%)$ pRErm 46 
plasmids (Figures 1, 3), presumably because of recombination between the duplicated IS6100s (Figure 4). Similar rearrangements have been reported in other integrons carrying directly repeated IS6100 copies $(25,26)$. Confirming the predicted functionality of pRErm46's tetRA(33) determinant, pRErm46-positive isolates were resistant to tetracycline, in contrast to those carrying the $\Delta \mathrm{C} 1 \mathrm{I}-\mathrm{tet} R A(33)$ form of the resistance plasmid (Table). However, all $R$. equi isolates were susceptible to the semisynthetic tetracycline derivative doxycycline, regardless of pRErm46 plasmid carriage (Table). This finding is consistent with previous data on Corynebacterium glutamicum showing that TetA(33) does not confer substantial cross-resistance to doxycycline (23).

Whereas a $\triangle C 1 I-t e t R A(33)$ plasmid deletion was detected in only 1 of the older (2002-2011) MDR 2287 isolates, the deletion was found in 10 of the 18 pRErm46-positive clonal isolates recovered during 2012-2017 (Figure 1). Deleted pRErm46s are observed in each of the clonal radiations of the MDR 2287 population and coexist with complete plasmids in more basal branches (Figure 3), indicating increasing occurrence because of repeated independent deletion events. The deletion was detected in all of the genetically heterogeneous macrolide-only-resistant R. equi isolates and the MDR 2287 (isolate no. 171) recovered from the Louisiana farm during the same period. This finding supports the notion that the latter was the source from which pRErm 46 had spread to other locally prevalent $R$. equi genotypes in that particular farm.

\section{Discussion}

This study demonstrates that the increasing prevalence of $M R^{R} R$. equi since its emergence in the late 1990s-early 2000s in equine farms in the United States (11-14) is primarily caused by the spread of the recently identified MDR 2287 clone (15). The oldest characterized MDR 2287 isolate dates from 2002 and was recovered in Kentucky (15) (Figure 1), where the clone likely emerged after the implementation of mass macrolide/rifampin antibiotic prophylaxis in foals (10). Since then, R. equi MDR 2287 has been frequently transferred between geographically distant farms, presumably through carrier horses. Active exchange of $R$. equi populations, previously noted in our earlier study (20), is evident in the United States

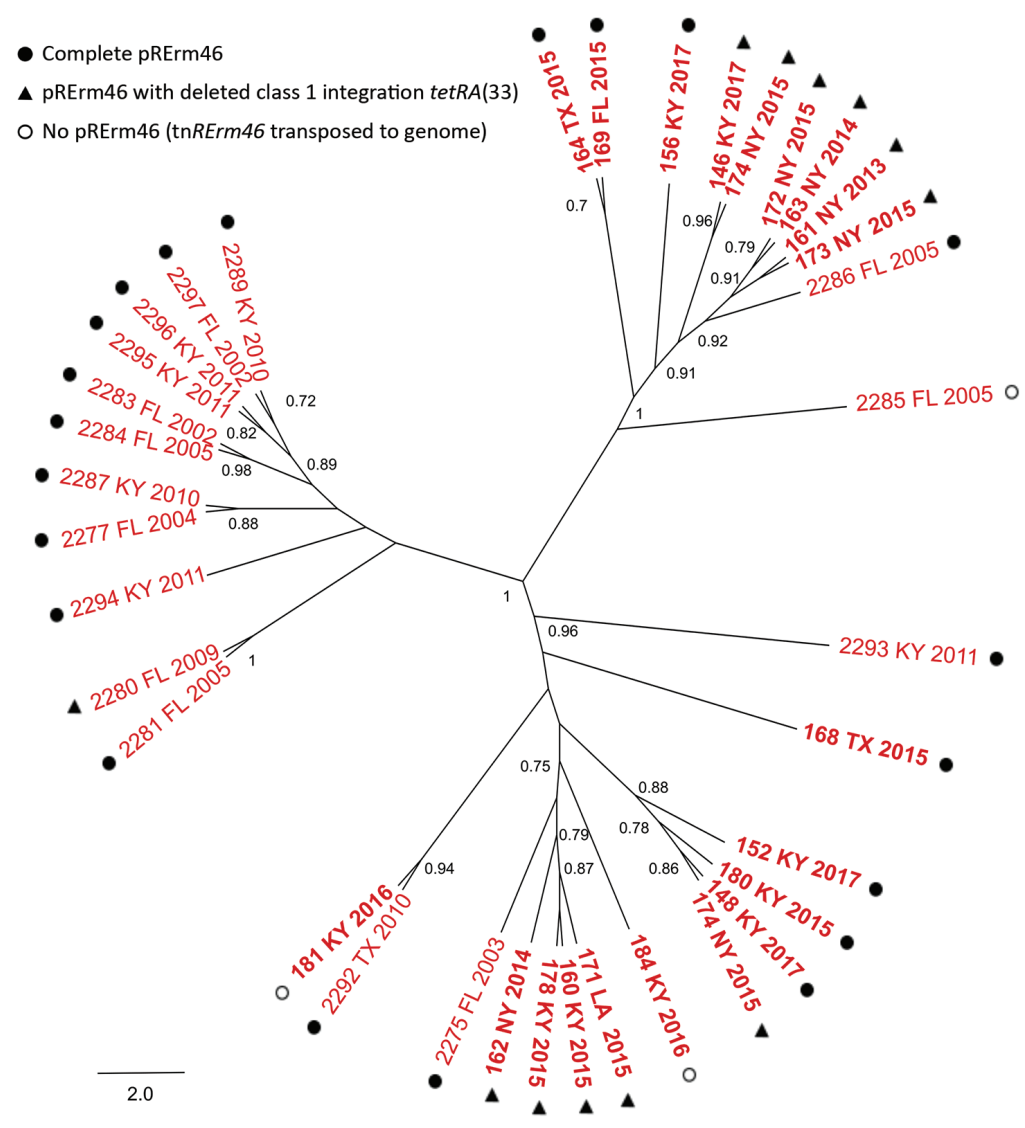

Figure 3. Phylogenetic population structure of multidrug-resistant Rhodococcus equi clonal complex 2287, United States. ParSNP core-genome tree of multidrug-resistant 2287 isolates shown in Figure 1. Nodes indicate bootstrap support for 1,000 replicates (values $>0.7$ shown). Tip labels indicate strain name, source (US state), and year of isolation. 
Figure 4. Schematic of $\triangle \mathrm{C} 1 \mathrm{l}$-tetRA(33) deletion in Rhodococcus equi pRErm46 macrolide resistance plasmid. Top bar shows full-size plasmid with the TnRErm46 transposon carrying the macrolideresistance erm(46) gene (in red, represented at nt position 32,567 [pRErm46 (PAM 2287) coordinates] common to all pRErm46 plasmids; additional TnRErm46 copies generated by transposition from original insertion may be present) and class 1 integron ( $\mathrm{C} 1 \mathrm{I}$, in yellow) with associated tetRA(33) tetracycline-resistance cassette (peach). Bottom bar shows pRErm46 plasmid with the $\triangle \mathrm{C} 1 \mathrm{l}$-tetRA(33) deletion. The deletion likely occurs through double crossover between the directly repeated flanking IS6100 sequences (dotted double arrow). Álvarez-Narváez et al. (15) includes detailed descriptions of pRErm46 plasmid and TnRErm46 transposon. and internationally when considering the phylogenetic tree in Figure 1. For example, the strains recovered from the Louisiana farm in this study are essentially identical to others found elsewhere in the United States. Also, terminal branches of the R. equi tree contain nearly identical equine isolates from different countries (e.g., the United States, France, and the Netherlands, or, in another case, Canada, Hungary, Sweden, and the United States) (Figure 1).

Despite the diversity of $R$. equi genotypes that typically circulate in farms $(21,22)$, the highly horizontally transferable erm(46) (TnRErm46) determinant remains largely confined to MDR 2287. This paradoxical clonal restriction is the probable consequence of the simultaneous requirement for erm(46) and the rpoB mutation under dual macrolide/rifampin pressure. More specifically, the clonal restriction is likely determined by the low odds of pRErm46/TnRErm46 and a high-resistance rpoB mutation (such as Ser531Phe in MDR 2287 or Ser531Tyr in MDR G2016) being acquired concurrently, and the latter effectively linking the mobile erm(46) determinant to a specific chromosomal background (15).
This interpretation implies several predictions. First, under dual macrolide/rifampin pressure, spread of an existing $\mathrm{MR}^{\mathrm{R}}$ strain through horse movements is more likely to contribute to the bulk of resistance than the generation of new $\mathrm{MR}^{\mathrm{R}}$ strains (15). Second, continued macrolide/rifampin therapy might eventually lead to the emergence of new $M^{R}$ clones, such as G2016 identified in this study, detected in 2016 in Kentucky and characterized by a novel rpoB $B^{5331 Y}$ mutation. Third, and importantly, if dual macrolide/rifampin selection ceases, unrestricted pRErm46/TnRErm46 horizontal transfer to other $R$. equi strains might occur. Our data appear to support these 3 possibilities.

The first and second scenarios are expected in horse-breeding areas such as Kentucky, Texas, or Florida, where R. equi is endemic and macrolide/ rifampin antibiotic prophylaxis has been commonly practiced $(10,27,28)$. Less intensive and more targeted antibiotic therapy is more likely in areas with smaller horse populations such as Louisiana (29), where pRErm46 spillover outside the MDR 2287 clone was detected (the third scenario). We hypothesize that a less intensive antibiotic

\footnotetext{
Table. Effect of absence of tetRA(33) determinant from pRErm46 plasmid on $R$. equi susceptibility to tetracycline and doxycycline, determined on macrolide-resistant isolates collected during $2012-2017^{*}$

\begin{tabular}{|c|c|c|c|c|}
\hline \multirow[b]{2}{*}{ Antibiotic } & \multicolumn{2}{|c|}{ pRErm46 } & \multicolumn{2}{|c|}{ pRErm46 $\Delta$ C1I-tetRA(33) } \\
\hline & Phenotype & $\mathrm{MIC}, \mu \mathrm{g} / \mathrm{mL} \neq$ & Phenotype & $\mathrm{MIC}, \mu \mathrm{g} / \mathrm{mL}$ \\
\hline Tetracycline & Resistant (100)§ & $21.33(8-48) \rrbracket$ & Susceptible (100)§ & $1.97(0.38-3) \pi$ \\
\hline Doxycycline & Susceptible (100) & $3.35(0.75-6)^{\star *}$ & Susceptible (100) & $1.06(0.25-3)^{* *}$ \\
\hline \multicolumn{5}{|c|}{$\begin{array}{l}\text { *Susceptibility data to other relevant antimicrobials are shown in Appendix Table } 2 \text { (https://wwwnc.cdc.gov/EID/article/27/2/20-3030-App1.pdf). } \\
\text { †Determined by disk diffusion technique. Isolate percentage shown in parenthesis. Zone diameter susceptibility breakpoints based on Clinical and } \\
\text { Laboratory Standards Institute interpretive criteria for Staphylococcus aureus, routinely used for } R \text {. equi susceptibility testing in the absence of specif } \\
\text { approved criteria for this species }(11,16) \text {. } \\
\text { †Minimal inhibitory concentration determined using Etest strips. Mean value (range in parenthesis). } \\
\$ p<0.001 \text { by } \chi^{2} \text { test. } \\
\prod_{* \star}^{*}<0.001 \text { by t-test. } \\
\text { Laboratory Standards Institute susceptibility breakpoint for doxycycline (susceptible } \leq 4 \mu \mathrm{g} / \mathrm{mL} \text {, intermediate } 8 \mu \mathrm{g} / \mathrm{mL}, \mathrm{resistant} \geq 16 \mu \mathrm{g} / \mathrm{mL} \text { ). }\end{array}$} \\
\hline
\end{tabular}
}


pressure, perhapsinvolving macrolidemonotherapy or a macrolide in combination with non-rifampin antibiotic drugs, disrupted the linkage between erm (46) and $r p o B^{\mathrm{s} 531 \mathrm{~F}}$ in the MDR 2287 strain found in the Louisiana farm, enabling the transfer of the plasmid to other locally prevalent $R$. equi strains (Figure 1).

Our analyses show that MDR 2287 has diversified since its first documented isolation into a clonal complex with several radiations (Figure 3). We also detected signs of microevolution in pRErm46, with a substantial rate of deletion of the C1I-tetRA(33) region in the 2012-2017 macrolide-resistant $R$. equi cohort, resulting in loss of tetracycline resistance. The clinical significance of this finding is unclear because tetracyclines are not used to treat $R$. equi infections in foals. An exception is doxycycline, which, because of its higher oral bioavailability in foals, greater tissue penetration, and better activity against gram-positive bacteria, might be used in cases of macrolide intolerance (or resistance) $(2,8,30)$. However, our data indicate that the pRErm46-encoded TetA33 does not confer clinically relevant cross-resistance to this semisynthetic tetracycline derivative. Genetic dispensability due to lack of antibiotic selection or fitness advantage might therefore be the likely reason for the increasing occurrence of $\Delta$ C1I-tetRA(33) pRErm46 plasmids in the macrolide-resistant $R$. equi population.

MDR R. equi shows resistance to several clinically relevant antibiotic drugs, including macrolides, lincosamides; streptogramins, and, in a substantial proportion, also tetracycline, all conferred by the pRErm 46 conjugative plasmid; and rifampin conferred by a chromosomal $r p o B^{\mathrm{S} 531 \mathrm{~F} / \mathrm{Y}}$ mutation. MDR R. equi also demonstrates intrinsic resistance to chloramphenicol (Appendix Table 2), which is often observed in R. equi. All of these antibiotic drugs are listed as critically or highly important for human medicine by the World Health Organization (31). Around 9\% of human R. equi infections are caused by equine-derived (pVAPApositive) strains, and about half of human cases are caused by porcine-derived (pVAPB-positive) isolates (5), which recent in vitro data demonstrate can also acquire pRErm46 (32). Therefore, in addition to compromising the therapeutic management of equine $R$. equi infection, these isolates represent a potential hazard to human health because of the risk of zoonotic transmission (or horizontal spread of the pRErm 46 resistance plasmid to other pathogens, either directly or through environmental microbiota [32]).
Although our study is not systematic and therefore probably underestimates the extent of MDR $R$. equi spread, our results provide valuable insight into the determinants underlying its emergence and dissemination. The data suggest a pattern of MDR $R$. equi spread and evolution directly determined by antibiotic pressure in equine farms. The stable therapeutic regimen applied over years for $R$. equi facilitates a unique understanding of the factors affecting the generation and evolution of MDR clones, and specifically how combination therapy might help in limiting the horizontal transfer of resistance. Although MDR R. equi is, to our knowledge, still limited to the equine population in the United States, our data predict a scenario of international spread through horse movements, indicating the need for interventions to control its dissemination and potential zoonotic transmission.

\section{Acknowledgments}

We thank L. Berghaus and A. Hanafi for technical support, L. Huber for help with the R commands, and G. Okem for preliminary experiments as part of a summer project.

This work was supported by grants from the Morris Animal Foundation (project no. D18EQ-401), the Hodgson Chair of Equine Research endowment of University of Georgia, the Link Equine Research Endowment at Texas A\&M University, and Horserace Betting Levy Board, UK (project no. prj796 to JV-B).

S.A.-N., S.G., N.C. and J.V.-B. designed the study. S.A.-N. performed the research. N.S. and N.C. collected isolates and susceptibility data. S.A.-N. and J.V.-B. analyzed and interpreted the data. J.V.-B. conceptualized the findings. S.A.-N. and J.V-.B. wrote the article.

New R. equi genome assemblies were deposited in GenBank under the accession numbers indicated in Appendix Table 1.

\section{About the Author}

Dr. Álvarez-Narváez is a clinical assistant professor at the University of Georgia. Her primary research interests include antimicrobial resistance mechanisms and host-pathogen interactions at the molecular level.

\section{References}

1. Prescott JF. Rhodococcus equi: an animal and human pathogen. Clin Microbiol Rev. 1991;4:20-34. https://doi.org/10.1128/ CMR.4.1.20

2. Vázquez-Boland JA, Giguère S, Hapeshi A, MacArthur I, Anastasi E, Valero-Rello A. Rhodococcus equi: the many facets of a pathogenic actinomycete. Vet Microbiol. 2013;167:9-33. https://doi.org/10.1016/j.vetmic.2013.06.016 
3. Yamshchikov AV, Schuetz A, Lyon GM. Rhodococcus equi infection. Lancet Infect Dis. 2010;10:350-9. https:/ / doi.org/ 10.1016/S1473-3099(10)70068-2

4. MacArthur I, Anastasi E, Alvarez S, Scortti M, VázquezBoland JA. Comparative genomics of Rhodococcus equi virulence plasmids indicates host-driven evolution of the vap pathogenicity island. Genome Biol Evol. 2017;9:1241-7. https://doi.org/10.1093/gbe/evx057

5. Ocampo-Sosa AA, Lewis DA, Navas J, Quigley F, Callejo R, Scortti M, et al. Molecular epidemiology of Rhodococcus equi based on traA, vapA, and vapB virulence plasmid markers. J Infect Dis. 2007;196:763-9. https:/ / doi.org/10.1086/519688

6. Vázquez-Boland JA, Meijer WG. The pathogenic actinobacterium Rhodococcus equi: what's in a name? Mol Microbiol. 2019;112:1-15. https:// doi.org/10.1111/ mmi.14267

7. Muscatello G, Leadon DP, Klayt M, Ocampo-Sosa A, Lewis DA, Fogarty U, et al. Rhodococcus equi infection in foals: the science of 'rattles'. Equine Vet J. 2007;39:470-8. https:/ / doi.org/10.2746/042516407X209217

8. Giguère $S$. Treatment of infections caused by Rhodococcus equi. Vet Clin North Am Equine Pract. 2017;33:67-85. https:/ / doi.org/10.1016/j.cveq.2016.11.002

9. Giguère $S$, Cohen ND, Chaffin MK, Slovis NM, Hondalus MK, Hines SA, et al. Diagnosis, treatment, control, and prevention of infections caused by Rhodococcus equi in foals. J Vet Intern Med. 2011;25:1209-20. https:// doi.org/10.1111/ j.1939-1676.2011.00835.x

10. Burton AJ, Giguère S, Sturgill TL, Berghaus LJ, Slovis NM, Whitman JL, et al. Macrolide- and rifampin-resistant Rhodococcus equi on a horse breeding farm, Kentucky, USA. Emerg Infect Dis. 2013;19:282-5. https://doi.org/10.3201/ eid1902.121210

11. Giguère S, Lee E, Williams E, Cohen ND, Chaffin MK, Halbert $\mathrm{N}$, et al. Determination of the prevalence of antimicrobial resistance to macrolide antimicrobials or rifampin in Rhodococcus equi isolates and treatment outcome in foals infected with antimicrobial-resistant isolates of R equi. J Am Vet Med Assoc. 2010;237:74-81. https://doi.org/ 10.2460/javma.237.1.74

12. Huber L, Giguère S, Slovis NM, Carter CN, Barr BS, Cohen ND, et al. Emergence of resistance to macrolides and rifampin in clinical isolates of Rhodococcus equi from foals in central Kentucky, 1995 to 2017. Antimicrob Agents Chemother. 2018;63:e01714-18. https://doi.org/10.1128/ AAC.01714-18

13. Anastasi E, Giguère $S$, Berghaus LJ, Hondalus MK, Willingham-Lane JM, MacArthur I, et al. Novel transferable erm(46) determinant responsible for emerging macrolide resistance in Rhodococcus equi. J Antimicrob Chemother. 2015;70:3184-90.

14. Erol E, Locke S, Saied A, Cruz Penn MJ, Smith J, Fortner J, et al. Antimicrobial susceptibility patterns of Rhodococcus equi from necropsied foals with rhodococcosis. Vet Microbiol. 2020;242:108568. https:/ / doi.org/10.1016/ j.vetmic. 2019.108568

15. Álvarez-Narváez S, Giguère S, Anastasi E, Hearn J, Scortti M, Vázquez-Boland JA. Clonal confinement of a highly mobile resistance element driven by combination therapy in Rhodococcus equi. MBio. 2019;10:e02260-19. https://doi.org/10.1128/mBio.02260-19

16. Berghaus LJ, Giguère S, Guldbech K, Warner E, Ugorji U, Berghaus RD. Comparison of Etest, disk diffusion, and broth macrodilution for in vitro susceptibility testing of Rhodococcus equi. J Clin Microbiol. 2015;53:314-8. https://doi.org/10.1128/JCM.02673-14
17. Koren S, Walenz BP, Berlin K, Miller JR, Bergman NH, Phillippy AM. Canu: scalable and accurate long-read assembly via adaptive $k$-mer weighting and repeat separation. Genome Res. 2017;27:722-36. https://doi.org/ $10.1101 /$ gr.215087.116

18. Treangen TJ, Ondov BD, Koren S, Phillippy AM. The Harvest suite for rapid core-genome alignment and visualization of thousands of intraspecific microbial genomes. Genome Biol. 2014;15:524. https:// doi.org/10.1186/s13059-014-0524-x

19. Price MN, Dehal PS, Arkin AP. FastTree 2-approximately maximum-likelihood trees for large alignments. PLoS One. 2010;5:e9490. https:// doi.org/10.1371/journal.pone.0009490

20. Anastasi E, MacArthur I, Scortti M, Alvarez S, Giguère S, Vázquez-Boland JA. Pangenome and phylogenomic analysis of the pathogenic actinobacterium Rhodococcus equi. Genome Biol Evol. 2016;8:3140-8. https://doi.org/10.1093/gbe/ evw222

21. Cohen ND, Smith KE, Ficht TA, Takai S, Libal MC, West BR, et al. Epidemiologic study of results of pulsed-field gel electrophoresis of isolates of Rhodococcus equi obtained from horses and horse farms. Am J Vet Res. 2003;64:153-61. https://doi.org/10.2460/ajvr.2003.64.153

22. Morton AC, Begg AP, Anderson GA, Takai S, Lämmler C, Browning GF. Epidemiology of Rhodococcus equi strains on Thoroughbred horse farms. Appl Environ Microbiol. 2001;67:2167-75. https:/ / doi.org/10.1128/ AEM.67.5. 2167-2175.2001

23. Tauch A, Götker S, Pühler A, Kalinowski J, Thierbach G. The 27.8-kb R-plasmid pTET3 from Corynebacterium glutamicum encodes the aminoglycoside adenyltransferase gene cassette aadA9 and the regulated tetracycline efflux system Tet 33 flanked by active copies of the widespread insertion sequence IS6100. Plasmid. 2002;48:117-29. https:// doi.org/10.1016/S0147-619X(02)00120-8

24. Chopra I, Roberts M. Tetracycline antibiotics: mode of action, applications, molecular biology, and epidemiology of bacterial resistance. Microbiol Mol Biol Rev. 2001;65:232-60. https:// doi.org/10.1128/MMBR.65.2.232-260.2001

25. Targant H, Doublet B, Aarestrup FM, Cloeckaert A, Madec JY. IS6100-mediated genetic rearrangement within the complex class 1 integron In104 of the Salmonella genomic island 1. J Antimicrob Chemother. 2010;65:1543-5. https://doi.org/10.1093/jac/dkq163

26. Partridge SR, Recchia GD, Stokes HW, Hall RM. Family of class 1 integrons related to In4 from Tn1696. Antimicrob Agents Chemother. 2001;45:3014-20. https:// doi.org/ 10.1128/AAC.45.11.3014-3020.2001

27. Huber L, Giguère S, Cohen ND, Slovis NM, Hanafi A, Schuckert A, et al. Prevalence and risk factors associated with emergence of Rhodococcus equi resistance to macrolides and rifampicin in horse-breeding farms in Kentucky, USA. Vet Microbiol. 2019;235:243-7. https://doi.org/10.1016/ j.vetmic.2019.07.010

28. Álvarez-Narváez S, Berghaus LJ, Morris ERA, Willingham-Lane JM, Slovis NM, Giguere S, et al. A common practice of widespread antimicrobial use in horse production promotes multi-drug resistance. Sci Rep. 2020;10:911. https://doi.org/10.1038/s41598-020-57479-9

29. Kilby ER. The demographics of the US equine population. In: Salem DJ, Rowan AN, editors. The state of the animals. Washington (DC): Human Society Press; 2007. p. 175-205.

30. Womble A, Giguère S, Lee EA. Pharmacokinetics of oral doxycycline and concentrations in body fluids and bronchoalveolar cells of foals. J Vet Pharmacol Ther. 2007;30:187-93. https://doi.org/10.1111/ j.1365-2885.2007.00857.x 
31. Collignon PJ, Conly JM, Andremont A, McEwen SA, Aidara-Kane A, Agerso Y, et al.; World Health Organization Advisory Group, Bogotá Meeting on Integrated Surveillance of Antimicrobial Resistance. World Health Organization ranking of antimicrobials according to their importance in human medicine: a critical step for developing risk management strategies to control antimicrobial resistance from food animal production. Clin Infect Dis. 2016;63:1087-93. https:/ / doi.org/10.1093/ cid/ciw475

32. Álvarez-Narváez S, Giguère S, Berghaus LJ, Dailey C, Vázquez-Boland JA. Horizontal spread of Rhodococcus equi macrolide resistance plasmid pRErm 46 across environmental Actinobacteria. Appl Environ Microbiol. 2020;86:e00108-20. https:/ / doi.org/10.1128/AEM.00108-20
33. Letek M, González P, Macarthur I, Rodríguez H, Freeman TC, Valero-Rello A, et al. The genome of a pathogenic Rhodococcus: cooptive virulence underpinned by key gene acquisitions. PLoS Genet. 2010;6:e1001145. https://doi.org/10.1371/journal.pgen.1001145

Address for correspondence: Sonsiray Álvarez-Narváez, College of Veterinary Medicine, Department of Large Animal Medicine, 2200 College Station Rd, Athens, GA 30602, USA; email: Sonsiray. Alvarez@uga.edu; or José A. Vázquez-Boland, Microbial Pathogenesis Laboratory, Edinburgh Medical School (Biomedical Sciences-Infection Medicine), Edinburgh BioQuater, Chancellor's Building, Edinburgh EH16 4SB, Scotland, UK; email: v.boland@ed.ac.uk

\section{August 2020}

\section{Parasitic Infections}

- Association of Dengue Virus and Leptospira Co-Infections with Malaria Severity

- US CDC Real-Time Reverse Transcription PCR Panel for Detection of Severe Acute Respiratory Syndrome Coronavirus 2

- Investigation and Serologic FollowUp of Contacts of an Early Confirmed Case-Patient with COVID-19, Washington, USA

- Characteristics and Outcomes of Coronavirus Disease Patients under Nonsurge Conditions, Northern California, USA, March-April 2020

- Tuberculosis in Internationally Displaced Children Resettling in Harris County, Texas, USA, 2010-2015

- Epidemiology of Legionnaires' Disease, Hong Kong, China, 2005-2015

- Rise in Babesiosis Cases, Pennsylvania, USA, 2005-2018

- Sporadic Creutzfeldt-Jakob Disease among Physicians, Germany, 1993-2018

- Population Genomic Structure and Recent Evolution of Plasmodium knowlesi, Peninsular Malaysia

- Analysis of MarketScan Data for Immunosuppressive Conditions and Hospitalizations for Acute Respiratory Illness, United States

- CrAssphage as a Novel Tool to Detect Human Fecal Contamination on Environmental Surfaces and Hands

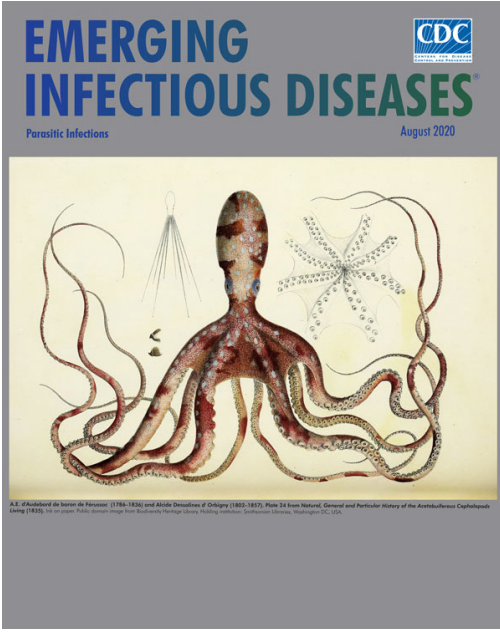

- Evaluating the Effectiveness of Social Distancing Interventions to Delay or Flatten the Epidemic Curve of Coronavirus Disease

- Presence of Segmented Flavivirus Infections in North America

- Human Outbreak of Trichinellosis Caused by Trichinella papuae Nematodes, Central Kampong Thom Province, Cambodia

- Increased Sensitivity of Plasmodium falciparum to Artesunate/Amodiaquine Despite 14 Years as First-Line Malaria Treatment, Zanzibar

- Factors Associated with Prescription of Antimicrobial Drugs for Dogs and Cats, United Kingdom, 2014-2016
- Linezolid-Associated Neurologic Adverse Events in Patients with MultidrugResistant Tuberculosis, France

- Naturally Acquired Human Plasmodium cynomolgi and P. knowlesi Infections, Malaysian Borneo

- Characterizing Norovirus Transmission from Outbreak Data, United States

- Imported Monkeypox, Singapore

- Population-Based Estimates of Chronic Conditions Affecting Risk for Complications from Coronavirus Disease, United States

- Prolonged Persistence of SARS-CoV-2 RNA in Body Fluids

- Prognostic Value of Leukocytosis and Lymphopenia for Coronavirus Disease Severity

- SARS-CoV-2 Phylogenetic Analysis, Lazio Region, Italy, FebruaryMarch 2020

- Plasma-Derived Extracellular Vesicles as Potential Biomarkers in Heart Transplant Patient with Chronic Chagas Disease

- Increasing Malaria Parasite Clearance Time after Chloroquine Therapy, South

- Disseminated Echinococcus multilocularis Infection without Liver Involvement in Child, Canada, 2018

- Canine Dracunculus Nematode Infection, Toledo, Spain

- Coronavirus Disease Outbreak in Call Center, South Korea

\section{EMERGING INFECTIOUS DISEASES}

To revisit the August 2020 issue, go to: https: / / wwwnc.cdc.gov/eid/articles/issue/ 26/8/table-of-contents 